\title{
THE TRITERPENOIDS OF SOME SPECIES OF RHODODENDRON
}

\author{
N. V. Belova and G. A. Fokina
}

Khimiya Prirodnykh Soedinenii, Vol. 6, No. 1, pp. 137-138, 1970

UDC 547.913

We have studied seven species of Rhododendron, family Ericaceae growing in the territory of the Soviet Union.

\begin{tabular}{|c|c|c|c|c|c|}
\hline \multirow{2}{*}{ Species } & \multirow{2}{*}{ Substance } & \multirow{2}{*}{$\mathrm{Mp},{ }^{\circ} \mathrm{C}$} & \multirow{2}{*}{ Derivative } & \multicolumn{2}{|r|}{$\mathrm{Mp},{ }^{\circ} \mathrm{C}$} \\
\hline & & & & found & literature data \\
\hline \multirow{2}{*}{$\begin{array}{l}\text { Rhododendron } \\
\text { adamsil } R \text { e hd. }\end{array}$} & Ursolic acid & 288 & Methyl ursolate & 170 & $170-171.5[1]$ \\
\hline & Oleanolic acid & 301 & Methyl oleanolate & 198 & $201[1]$ \\
\hline \multirow[t]{2}{*}{$\begin{array}{l}\text { Rhododendron au- } \\
\text { reum Georg. }\end{array}$} & Campanullin & 204 & $\begin{array}{l}\text { Alnus-5 }(10) \text {-enol } \\
\text { Alnus- } 5(10) \text {-enol acetate }\end{array}$ & $\begin{array}{l}237 \\
284\end{array}$ & $\begin{array}{l}242[2,3] \\
302[2,3]\end{array}$ \\
\hline & Taraxerone & 242 & $\begin{array}{l}\text { Taraxerone } 2,4 \text {-dinitro- } \\
\text { phenylhydrazone }\end{array}$ & 256 & $257-258.5[4]$ \\
\hline \multirow{2}{*}{$\begin{array}{l}\text { Khododendron da- } \\
\text { huricum L. }\end{array}$} & Taraxerol & 272 & Taraxerone & 242 & $245-249[1]$ \\
\hline & $\begin{array}{l}\text { Betulin } \\
\text { Ursolic acid }\end{array}$ & $\begin{array}{l}259 \\
286\end{array}$ & $\begin{array}{l}\text { Bet ulin acetate } \\
\text { Methyl ursolate }\end{array}$ & $\begin{array}{l}218 \\
168\end{array}$ & $\begin{array}{l}221[1] \\
170-171.5[1]\end{array}$ \\
\hline $\begin{array}{c}\text { Rhododendron kot- } \\
\text { schyi S i m k. }\end{array}$ & Uvaol & 222 & Uvaol acetate & 149 & $153-154.5[1]$ \\
\hline \multirow{4}{*}{$\begin{array}{l}\text { Rhododendron lu- } \\
\text { teum Swe t } \\
\text { Rhododendron muc- } \\
\text { ronulatum T } \mathrm{u} \text { c } z \text {. }\end{array}$} & Friedelin & 262 & $\begin{array}{l}\text { Uvaol benzoate } \\
\text { Friedelin oxime }\end{array}$ & $\begin{array}{l}104 \\
285\end{array}$ & $290[1]$ \\
\hline & Taraxerol & 280 & Taraxerol acetate & 296 & $303-305[1]$ \\
\hline & Simiarol & 209 & Simiarol acetate & 209 & $209[5,6]$ \\
\hline & & & $\begin{array}{l}\text { Simiarol benzoate } \\
\text { Simiarone }\end{array}$ & $\begin{array}{l}194 \\
205\end{array}$ & $207[5,6]$ \\
\hline & & & Episimiarol & 191 & $197[5]$ \\
\hline & & & $\begin{array}{l}\text { Isosimiarol } \\
\text { Isosimiarol acetate }\end{array}$ & 259 & $268[6]$ \\
\hline $\begin{array}{c}\text { Fhododendron } \\
\text { schlippenbachil } \\
\text { M a x i m. }\end{array}$ & Taraxerol & 278 & Taraxerol acetate & 300 & $303-305[1]$ \\
\hline
\end{tabular}

From chloroform extracts of leafy shoots of the plants by chromatography on alumina, a series of crystalline substances was isolated some of which were identified as campanullin, taraxerone, friedelin, simiarol, taraxerol, betulin, uvaol, and ursolic and oleanolic acids. Taraxerol was found in three species, and ursolic acid was isolated from two plants.

For the identification of all the compounds isolated, a number of derivatives was obtained by the usual methods. All the substances investigated were characterized on the basis of the results of IR spectroscopy. The triterpene compounds obtained and their derivatives that were identified from literature data, with the exception of campanullin and simiarol, gave no depression of the melting point with the corresponding authentic samples.

\section{R E F E R E N C ES}

1. T. G. Halsell and R. T. Aplin, Fortschritte der Chemie organischer Naturstoffe, 22, 170-191, 1964.

2. S. Rangaswami and K. Sambamurthy, Proc. Ind. Acad. Sci., A, 54, 132, 1961.

3. J. M. Beaton, F. S. Spring, R. Stevenson, and J. L. Stewart, Tetrah. , 2, 246, 1958.

4. M. G. Matyukhina, V. S. Shmukler, and A. A. Ryabinin, ZhOKh, 35, 579, 1965.

5. W. H. Hui, H. R. Arthur, and V. Angsusingh, Symposium on Phytochemistry, Hong Kong, $142-144,1961$. 6. R. T. Aplin, H. R. Arthur, and W. H. Hui, J. Chem. Soc. (C. Org.), 14, 1251, 1966. 